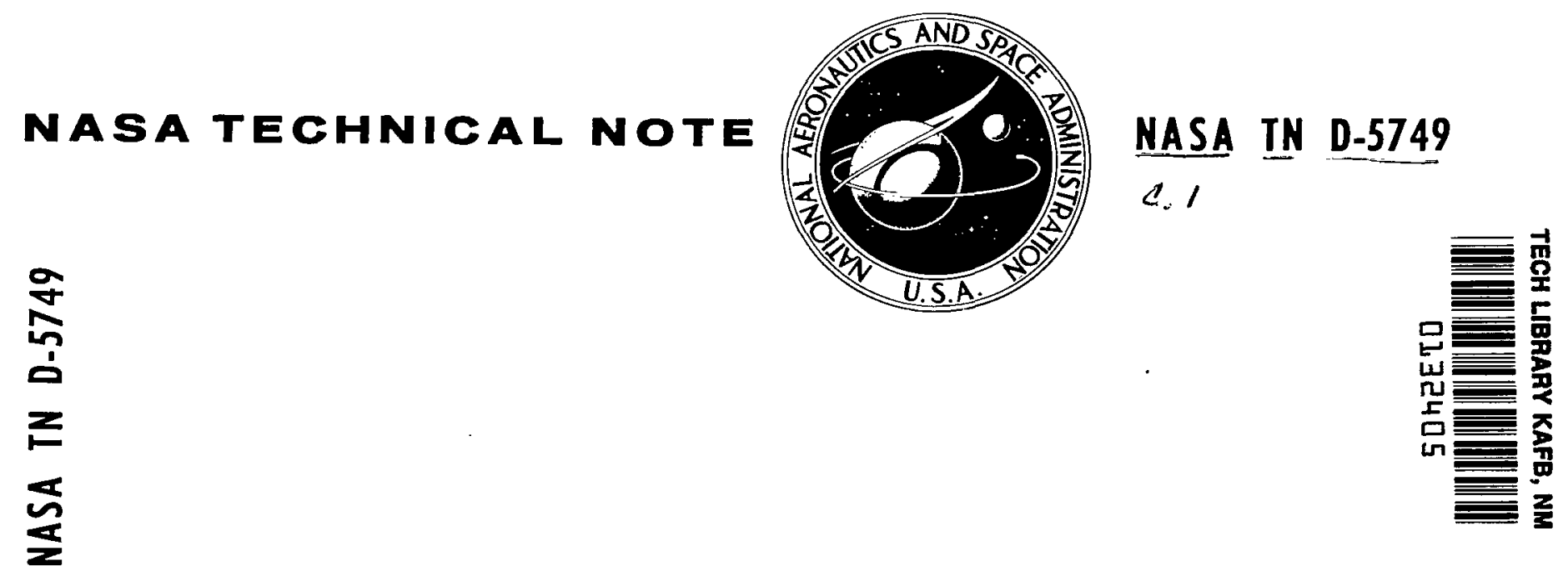

IOANY COPY: RETURN TO ANWL (WLOL)

RIRTIMND AFB, N MEX

\title{
INTERFEROGRAMS OF WINDOW WAVEFRONT
}

DEFORMATIONS AS A MEASURE

OF ANGULAR DEVIATIONS

TO A LINE OF SIGHT

by Thomas M. Walsh and David N. Warner, Jr.

Ames Research Center

Moffett Field, Calif.

NATIONAL AERONAUTICS AND SPACE ADMINISTRATION - WASHINGTON, D. C. - APRIL 1970 
1. Report No. NASA TN D-5749

2. Government Accession No.

4. Title and Subtitle

INTERFEROGRAMS OF WINDOW WAVE FRONT DEFORMATIONS AS A MEASURE OF ANGULAR DEVIATIONS TO A LINE OF SIGHT

7. Author(s)

Thomas M. Walsh and David N. Warner, Jr.

9. Performing Organization Name and Address NASA Ames Research Center Moffett Field, Calif. 94035

12. Sponsoring Agency Nome and Address

NATIONAL AERONAUTICS AND SPACE ADMINISTRATION

Washington, D. C., 20546
14. Sponsoring Ageney Code

3. Recipient's Cotalog No.

5. Report Date April 1970

6. Performing Organization Cade

8. Performing Organization Report No. A-3074

10. Work Unit No. 125-17-02-13-00-21

11. Contract or Grant No.

13. Type of Report and Period Covered TECHNICAL NOTE

Podo

15. Supplementary Notes

16. Abstract

\begin{abstract}
An interferogram of the wavefront deformation to a flat wave caused by an aircraft or spacecraft window contains all the error contribution effects of the window. The interferogram may be analyzed to determine the slope of this wavefront at a given point, which yields the angular deviation to a line of sight through the point. The interferogram as used in this study is a two-dimensional photo representing the three-dimensional relationship of the transmitted wave relative to the flat reference wave. The progression from light to dark fringes is the progression of the third dimension normal to the
photo, and the fringes are thus similar to contour lines on a topographic map. To demonstrate the ability to obtain interferophoto, and the fringes are thus similar to contour lines on a topographic map. To demonstrate the ability to obtain inter grams, to analyze interferograms, and to achieve sufficient accuracy, an aircraft window and spacecraft window were obtained in the laboratory and were also measured precisely by an autocollimator system. The standard deviation of the transmitted wave deviations derived from interfe rograms relative to directly measured deviations was 0.7 second of arc, indicating that the transmitted wave interferogram method is a good tool for window error determination.
\end{abstract}

17. Key Words Suggested by Author(s)

$$
\begin{aligned}
& \text { Interferometry } \\
& \text { Line-of-sight deviations } \\
& \text { Window (aperture) } \\
& \text { Wa vefront deformations } \\
& \text { Space navigation } \\
& \text { Sextant }
\end{aligned}
$$

19. Security Classif. (of this report) Unclassified
18. Distribution Statement

Unclassified - Unlimited

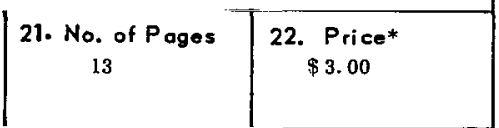

*For sale by the Clearinghouse for Federal Scientific and Technical Information Springfield, Virginia 22151 


\section{INTERFEROGRAMS OF WINDOW WAVEFRONT DEFORMATIONS AS}

\section{A MEASURE OF ANGULAR DEVIATIONS TO}

\section{A LINE OF SIGHT}

By Thomas M. Walsh and David N. Warner, Jr.

Ames Research Center

SUMMARY

An interferogram of the wavefront deformation to a flat wave caused by an aircraft or spacecraft window contains all the error contribution effects of the window. The interferogram may be analyzed to determine the slope of this wavefront at a given point, which yields the angular deviation to a line of sight through the point. The interferogram as used in this study is a two-dimensional photo representing the three-dimensional relationship of the transmitted wave relative to the flat reference wave. The progression from light to dark fringes is the progression of the third dimension normal to the photo, and the fringes are thus similar to contour lines on a topographic map. 'To demonstrate the ability to obtain interferograms, to analyze interferograms, and to achieve sufficient accuracy, an aircraft window and spacecraft window were studied in an experimental test. The deviations to a line of sight were derived from transmitted wave interferograms obtained in the laboratory and were also measured precisely by an autocollimator system. The standard deviation of the transmitted wave deviations derived from interferograms relative to directly measured deviations was 0.7 second of arc, indicating that the transmitted wave interferogram method is a good tool for window error determination.

\section{INTRODUCTION}

Because of the unparallelism of the pane surfaces, the bowing of the panes due to environmental pressures and temperatures, and anomalies in the glass, aircraft and spacecraft windows cause angular deviation errors in a line of sight passing through the glass of the windows. These errors directly affect navigation sextant measurements, such as those of NASA Experiment $\mathrm{T}-2$ on the Gemini GT-12 flight and those measurements made through windows of the NASA CV-990 research aircraft. The window error corrections for the T-2 experiment are found in reference 1 and were determined by direct measurement with a large aperture autocollimator. Aircraft window errors of the latter experiment are found in reference 2 and were determined by computation in a sophisticated ray trace program.

The advantages of the method described herein are its relative ease and dependability as compared with the two methods previously mentioned. The autocollimator method is a very time-consuming and difficult task, as 
reference 3 indicates (a minimum of 1200 measurements was made). Autocollimator measurements are prone to operator errors and equipment errors. In addition, the autocollimator is, in general, limited to examining only a few locations because of the time required for testing all possible apertures; consequently, some information is lost that may be desired at a later date. The ray trace method used in reference 2 necessitates the assumption of initial conditions. For a multipane window, such as a four-pane spacecraft window, the validity of the results would be only as good as the assumptions. If the window edge constraints are unknown, sophisticated interferometric techniques must be used to determine distortion of the window panes under pressure conditions before the line-of-sight deviations can be computed. The corrections in reference 2 were for a single-pane aircraft window.

The advantage of the interferometric method of this study is that it produces a permanent record of a wavefront after it has passed through any number of window panes, which may be influenced by (1) any combination of wedge angles; (2) index of refraction inhomogeneities (3) surface shapes distorted during manufacture; and (4) surface shapes distorted by environmental pressures, temperature differences, and mechanical forces due to undefined edge conditions. All apertures are covered by one exposure so no information is lost because of the press of time. The interferometric method is relatively easy compared with the autocollimator method in regard to the problems of apparatus setup, time expended, maintaining precision, operator fatigue, etc. The interferogram can be interrogated at any later date for window error, so only the specific aperture of interest need by examined at the time the original navigation data are reduced.

The purpose of this study was to investigate an interferometric method of determining window error corrections. The proposed method had two main steps: (1) a reference flat wavefront was introduced into a window under simulated space environmental conditions, and an interferogram of the existing distorted wavefront (hereafter called the transmitted wavefront) was obtained; (2) the interferogram was analyzed to obtain the window deviation errors. The purpose of this study was to implement and validate the method by showing that:

1. A reference flat wavefront may be made to enter a test window and a suitable transmitted wave interferogram may be obtained if the proper equipment, procedures, and techniques are used.

2. (a) The interferogram so obtained represents the distortion to a flat wavefront caused by the window. (b) The normal to the distorted wavefront is the direction of the wavefront at each coordinate point and, as such, is the direction of the deviated ray at each point.

3. The interferogram can be analyzed by procedures derived herein to obtain the deviation in seconds of arc.

4. Actual window deviations so determined compare closely with directly measured line-of-sight deviations for both a spacecraft window and an aircraft window. 
One spacecraft window and one aircraft window were investigated. The aircraft window was chosen for the study because it represented a lower qua1ity optical window that would yield high deviations. Thus the ability of the interferogram method could be tested for large as well as small deviations. The angular deviations to a line of sight were measured at several apertures and incidence angles by autocollimator in laboratory tests. Almost simultaneously, transmitted wave interferograms were also obtained. The interferograms were analyzed, deviations were computed from them, and the results were compared with the measured deviations. The objective of the test data was to show that the interferogram results were comparable to direct measurement results and, at the same time, demonstrate that laboratory optical equipment and the procedures and techniques used could generate an interferogram of sufficient fidelity.

The difficulties and limitations of the alternative methods mentioned for determining window errors provided the incentive for this study. The utilization of this interferogram analysis method is based, however, on the ability to obtain a transmitted wave interferogram of the window. Reference 4 describes a method for obtaining, through large glass panels, a transmitted wave measurement using a Fizeau interferometer. It is very simple but limited to windows that can be adapted to a Fizeau setup. Interferograms of Gemini windows were obtained as described in reference 3 . In the case of the Apollo window, which is potted into place in the Apollo spacecraft she11 and has an environmental pressure of about 5 psia inside the she11, the necessary interferograms would be obtained with some difficulty. Reference 5 points up some of the problems and solutions to interferometry in a hostile environment and is pertinent to obtaining interferograms of the Apollo window. While the actual physical adaptation of the interferometer to a particular task is very important to the use of the proposed method, this study was not intended to solve such specific problems.

\section{APPARATUS AND PROCEDURE}

The apparatus is basically optical in function and is similar to that used in reference 3 . However, the combination of components for obtaining

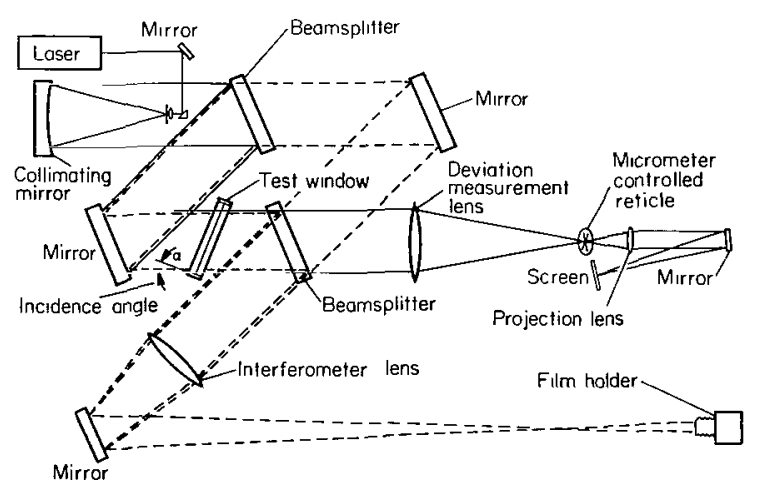

Figure 1.- Mach-Zender interferometer and deviation measurement system. practically simultaneously, interference patterns, autocollimator deviations, and photographic records of window aperture, window grid, and interference pattern is unique and is critical to the pursuit of this study. Figure 1 shows the schematic arrangement of the apparatus. The dashed lines indicate a ray trace for the interferogram elements and the solid lines indicate a ray trace for direct deviation measurement elements. We desired to obtain a precision of 1 second-of-arc, so it was important to keep the time interval for data 
acquisition to a minimum (15 minutes) to avoid temperature shifts and drift due to mechanical causes. Therefore, the test window was untouched between acquisition of interferograms and deviation data, and as many optical elements as possible were used in common with no critical element adjustment being necessary in the data acquisition time interval. The light source was a 632.8-nanometer He-Ne laser, which was focused into a long focal length, parabolic mirror to generate a highly collimated bundle of light. This bundle of light then entered a Mach-Zender interferometer containing the test window. Light from both paths of the interferometer was brought to focus at the film plate by the interferometer lens so that a wire grid on the window surface was in sharp focus at the film plate. Simulataneously, for the autocollimator measurements, some light through the window path of the interferometer was brought to focus by a deviation measurement lens on a reticle mounted on a $x-y$ cross slide. The light spot and reticle were in turn projected on a screen for precise setting of reticle on the spot image.

The procedure was to adjust the interferometer for zero fringes with the interferometer empty (i.e., without the window) and also to obtain an initial $x-y$ micrometer deviation reading with the crossbars on the focused laser spot. The window was then inserted to a desired incidence angle, and all data for that incidence angle were obtained before the window was rotated to a new incidence angle or a new plane of incidence.

Two windows were used in the test program: a "spacecraft" window, which represents high optical quality, and an "aircraft" window, which represents moderate optical quality. The high optical quality window is arbitrarily defined here as one producing less than 10-seconds-of-arc deviation to a line of sight, whereas moderate optical quality is arbitrarily defined as producing up to 40-seconds-of-arc deviation. This is almost more than could be tolerated from a window suitable for spacecraft or aircraft navigation measurements. The spacecraft window used two Gemini right-hand window panes mounted in a modified Gemini-type frame and pressurized to 14.7-psi gage between the panes. This was similar to a spacecraft environment and induced bowing of the panes to produce realistic distortion to a plane transmitted wave. The aircraft window had been installed in a Convair 990 aircraft used in scientific experiments at high altitudes. It was a single, thick pane of glass, mounted in the frame used in the aircraft.

By definition, the plane of incidence contains the incident ray and is perpendicular to the window. Figure 2 shows the orientation of the plane of incidence relative to the window as the angle $\theta_{0}$. The planes of incidence chosen for rays for which deviations were to be measured were parallel to the plane containing the horizontal centerline of the window. Figure 3 shows this line and the apertures along the vertical and horizontal centerlines. Thus, $\theta_{0}=0$ is the orientation chosen for the plane of incidence for the whole study. The origin of the coordinate system moves from aperture to aperture.

Angles of incidence of $15^{\circ}$ and $45^{\circ}(\alpha$ in fig. 2) were used. A wire grid on the face of the window was used to locate discrete points on the interferogram from photographs of its outline superimposed on the interferograms. One-inch-diameter holes in a mask were used as apertures for deviation measurement. The holes were centered on the grid intersections. 


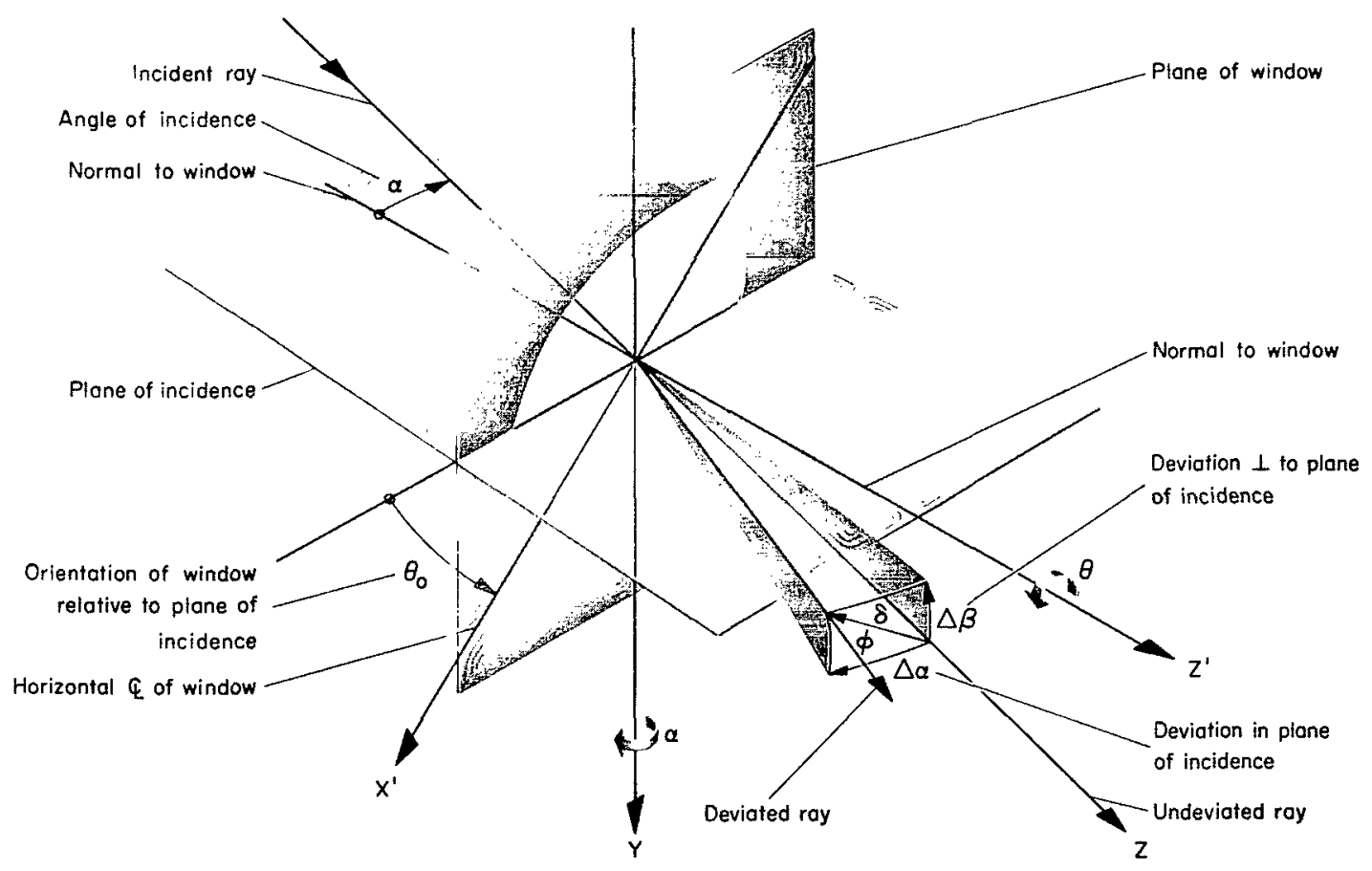

Figure 2.- Spacecraft window-ray coordinates.

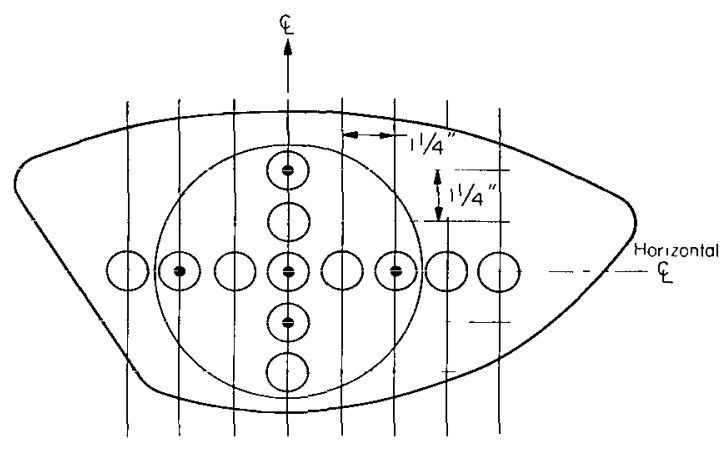

Figure 3.- Spacecraft window-aperture location.
It was important to know the precision of each of the two complete systems for data analysis. These two systems, the deviation measurement system and the interferometer imaging system, have many elements in common in the collimator and the Mach-Zender interferometer. These elements are of $1 / 10$ and $1 / 20$ wave conformity quality, which is mainly responsible for the net-system precisions. The line-of-sight deviation measurement system demonstrated a repeatability of readout for a sample data point of $1 \sigma$ (standard deviation) of 0.34 second of arc, and showed a maximum variation of 0.7 second of arc for al1 samples taken over its $12-i n c h$ aperture. This deviation precision bears testimony to the flatness of the wavefront generated in the collimator after passing through one path of the interferometer. Observations of the straightness of fringes generated by the interferometer indicated that the two wavefronts in the interferometer were flat and uniform to about 0.4 wavelength over the 12 -inch aperture. This figure is consistent with the 0.7 -second-of-arc deviation mentioned previously. Prior to use, the interferometer when empty was always adjusted so that no fringes were visible over the 12-inch aperture. This means that there was less than 1 wavelength initial bias across the interferometer aperture. 
The determination of deviations from interferograms is illustrated in figures 4 and 5 . The fringes represent equal distance contours of the transmitted wave from a reference (flat) wavefront. The more numerous spurious fringes indicated on figure 4 resulted from lens reflections that were

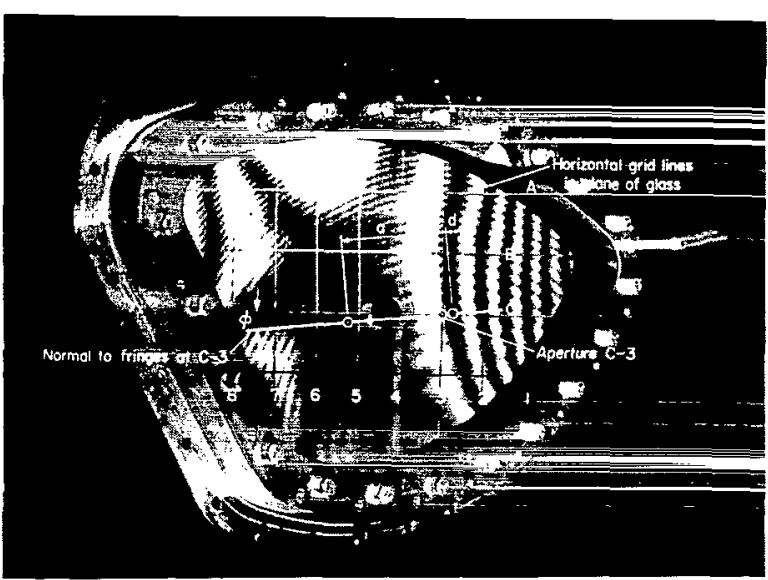

Figure 4.- Normal to fringes at aperture $\mathrm{C}-3$ indicating direction of line of sight.

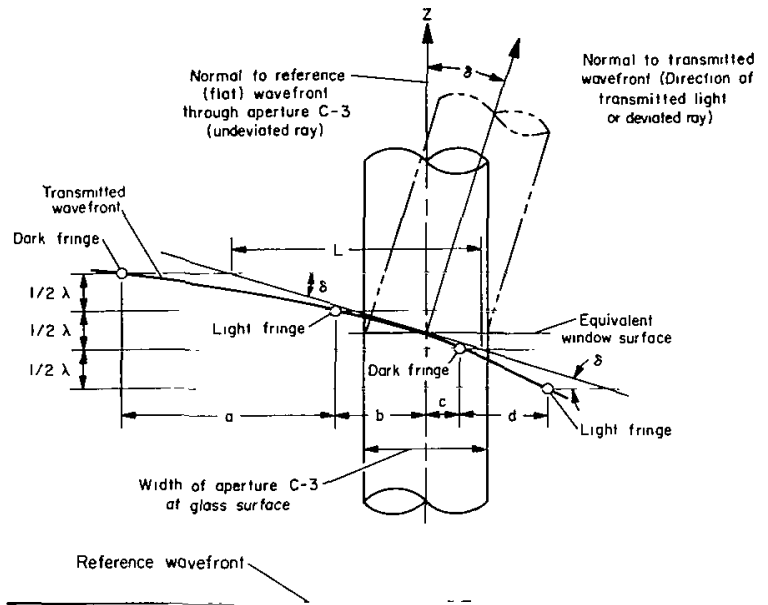

Figure 5.- Graphical construction of wavefront cross section at aperture $\mathrm{C}-3$.

eliminated from the aircraft window interferograms. The theory of interference fringes may be found in references 6 and 7 . A normal to the fringes at, say, aperture $\mathrm{C}-3$ (fig. 4) represents the trace of the plane containing the normal to the wavefront. The angle $\phi$ is the angle the trace makes with the horizontal grid lines. The spacing of fringes (dark to light) along this trace $a,(b+c)$, and $d$ as plotted in figure 5 recreates the wavefront in cross section. The normal at $\mathrm{C}-3$ to this cross section is the direction of advance of the transmitted wavefront at this point. The angle of interest, the deviation angle $\delta$ ( $f i g .2$ ), may be determined by:

$$
\tan \delta=\frac{\lambda M}{L R}
$$

for small angles

$$
\delta \cong \frac{\lambda M}{L R} \text { seconds of arc }
$$

where

$\delta \quad$ angle, seconds of arc

$\lambda \quad 632.8 \times 10^{-9} \mathrm{~m}$

$M \quad$ magnification used in readout of figure $4=1.7$ 
$\mathrm{R}$ radians per second of arc $=4.848 \times 10^{-6}$

$$
\delta=\frac{632.8 \times 10^{-9} \times 1.7}{4.848 \times 10^{-6} \times 0.04115}=5.4 \text { seconds of arc }
$$

The precision of this graphical technique of interferogram interpretation depends on the precision of the inputs into the linear equation used above for computing deviations. Repetitive interpretation of the interferogram to determine angular deviation of several apertures was performed with all error sources included in the measurements. Thus, a normal was erected to the fringes at an aperture of interest, measurements were made of fringe spacings, a plot was made, a normal was erected to the wavefront profile thus plotted, "L" was measured, and the angle $\delta$ was computed. This procedure was repeated several times at each aperture. Less than 1 second of arc difference in deviation was noted at any aperture. The angle of the trace plane was repeatable to $1^{\circ}$ for large deviation fringes. The components $\Delta \alpha$ and $\Delta \beta$ in figure 2 of the deviated ray $\delta$ lie along the horizontal and vertical grid lines of figure 4 and can be computed, provided $\delta$ and $\phi$ are known. An uncertainty of $1^{\circ}$ in $\phi$ will cause errors of less than 1 second of arc in the horizontal and vertical components of deviation for all values of $\delta$ less than 59 seconds of arc. All values of $\delta$ were well below this. In the instances of small deviation, where fringes were few and it was difficult to repeat the $\phi$ measurement to $1^{\circ}$, the total deviation was so low that the components of deviation were well below 1 second of arc.

When many apertures across a transmitted wavefront interferogram must be analyzed for deviations, a computational method, such as described in reference 8 , would prove to be useful. In the computational procedures of that report, a window surface model is described that yields a three-dimensional, least-squares, polynomial approximation to the surface. The procedure could be used to approximate the surface of the transmitted wave of this study. The unit vector normal to the surface at a given point could then be obtained by computation and would yield the deviation in seconds of arc from the original (reference) unit vector. This was not done in the study because of the limited number of apertures tested, but the application would be straightforward.

A transmitted wave interferogram, such as figure 6(b), could be indicative of either a diverging or a converging wavefront. (The dark vertical rod and ball in figure 6 is the support for part of the light source.) This ambiguity can be resolved by a second interferogram taken as in figure $6(a)$ or $6(\mathrm{c})$. The movement of the tangent spot of figure $6(\mathrm{~b})$ (closed fringe to the left of center) with rotation of the reference wavefront is the clue to the nature of the wavefront, as simply portrayed in the line diagram to the right of the photographs. Both figures $6(\mathrm{a})$ and (c) illustrate the spot motion for a convex wavefront. The spot motion would be opposite if the 

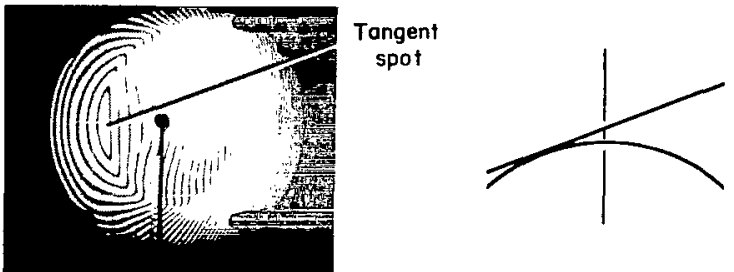

(a) Counterclockwise rotation of reference wavefront.
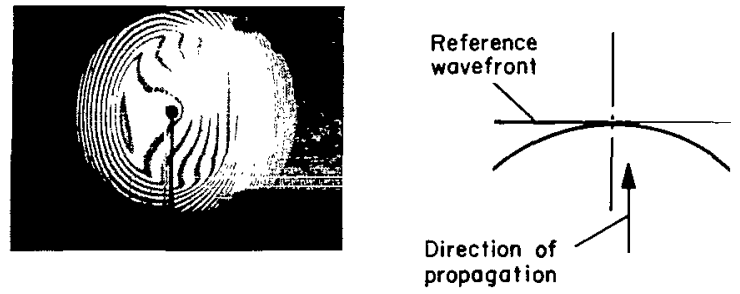

(b) Zero order.
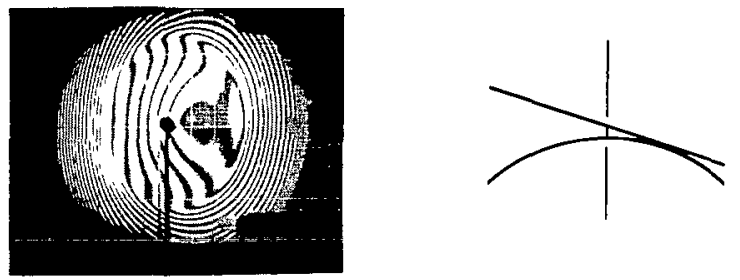

(c) Clockwise rotation of reference wavefront.

Figure 6.- Determination of convex wavefront. wavefront were converging. Thus, a study of movement of the tangent spot as one of the Mach-Zender interferometer mirrors is rotated with a thumbscrew reveals the nature of the wavefront.

\section{RESULTS}

The first step of the objectives, obtaining interferograms of a wavefront transmission through a window under environmental conditions, was accomplished as can be seen in figures 4, 6, 8,10 , and 11 , which were some of those obtained during the investigation. These figures are evidence of the successful adaptation of large aperture (12 inch) interferometer components to long optical path lengths (over $100 \mathrm{ft}$ ) to obtain interferograms.

The technique used in the procedure showed that the interferogram represents the distortion to a flat wavefront caused by the window. The initial adjustment, with the window out of the interferometer path, gave a field devoid of fringes. With insertion of the window, the fringes appeared as in the above photographs, and the usual inter-

pretation of the fringes could be made from the number and curvature, which are indicative of sphericity of the new wavefront. The fringe 1ines, as in the cross section of figure 5 , reproduce the three-dimensional wavefront in two dimensions. Figure 5 indicates that the direction of any deviated ray at a point is normal to such a wavefront at that point.

Using the interferograms and constructing cross sections, such as figures 4 and 5 , and the formula for $\tan \delta$ in the procedures, did yield deviations in seconds of arc. Thus, the concept of deriving deviations of a line of sight from interferograms was demonstrated.

The main purpose of the study was to show that interferometric deviations are precise enough to correct window deviation errors. The following results are pointed out to demonstrate that this was true.

1. The angular deviations through the spacecraft windows with the direct deviation measurement system at $15^{\circ}$ incidence are plotted in figure 7 . The results, by interferometric interpretation of figure 8 , agree very well with the direct measurements (to within 1 second of arc). The interference 


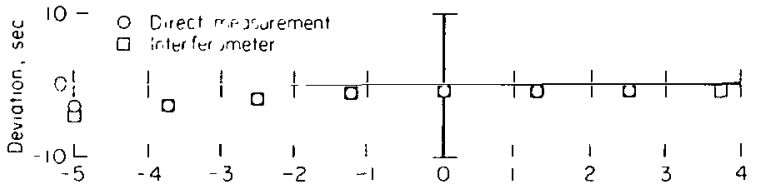

(a) Deviation in plane of incidence for apertures in horizontal centerline.

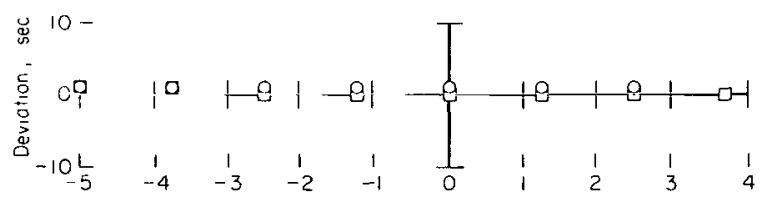

(b) Deviations perpendicular to plane of incidence for apertures in horizontal centerline.

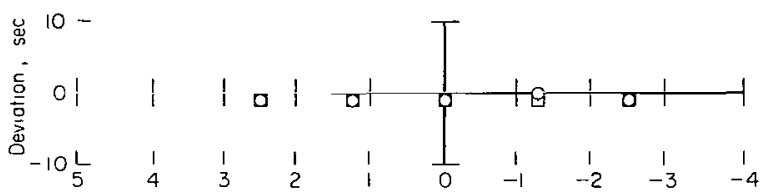

(c) Deviations in plane of incidence for apertures in vertical centerline.

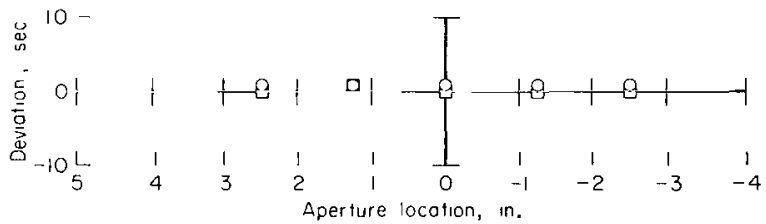

(d) Deviations perpendicular to plane of incidence for apertures in vertical centerline.

Figure 7.- Line-of-sight angular deviations through a spacecraft window at $15^{\circ}$ incidence.

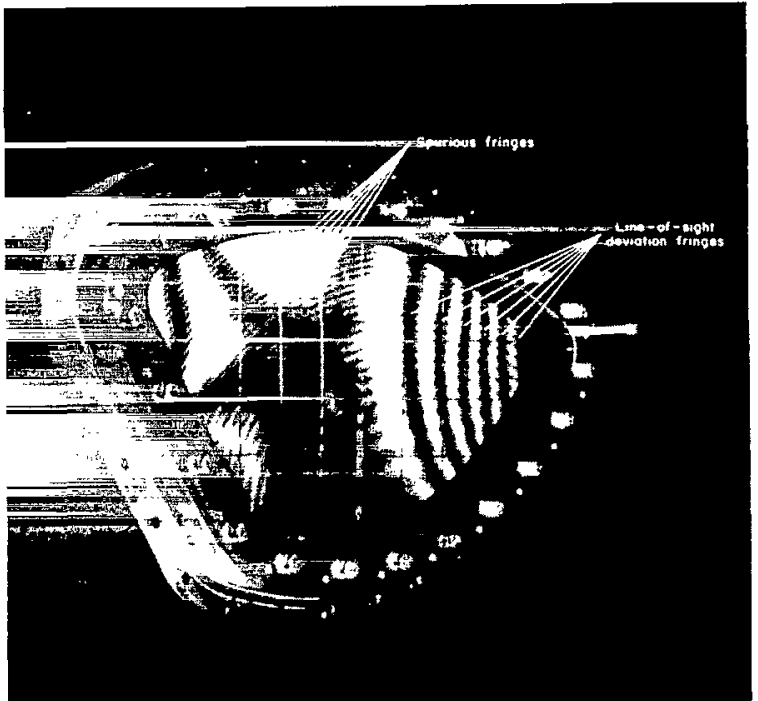

Figure 8.- Transmitted wave of spacecraft window at $15^{\circ}$ incidence.

measurements were made by interpretation of the transmitted wave interferogram shown in figure 8 where the line-of-sight deviation fringes are the large ragged dark areas as noted.

2. With an increase in angle of incidence to $45^{\circ}$ degrees, the agreement of direct measurement data with data obtained by interpretation of the interferogram was within 2 seconds of arc as shown in the data plots of figure 9 . The corresponding interferogram (fig. 10) shows an increase in fringes with this higher incidence. The maximum measured deviation increased to 12 seconds of arc.

3. The aircraft window, as can be seen from the interferogram of figure 11, has a high quality optical area centrally located, and moderate quality otherwise. The deviations reached a maximum of 34 seconds as plotted in figure 12. Again, the figure shows that the interferometric deviations practically superimposed on the measured value and the greatest difference was only 2 seconds of arc. The 1-inch-diameter aperture centered at grid intersection (c, 2-1/2 in.) of figure 11, is half on the high quality central portion of the window and half on the moderate quality portion. Distortion of the image observed by the direct measurement technique revealed this discontinuity in the transmitted wave; therefore, separate deviations were measured for each half of the aperture. The interferometric data extracted from each half of this aperture agreed closely (within $2 \mathrm{sec}$ ) with the measured data. 


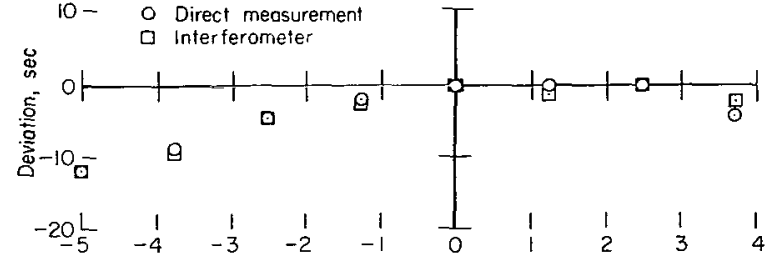

(a) Deviation in plane of incidence for apertures in horizontal centerline.

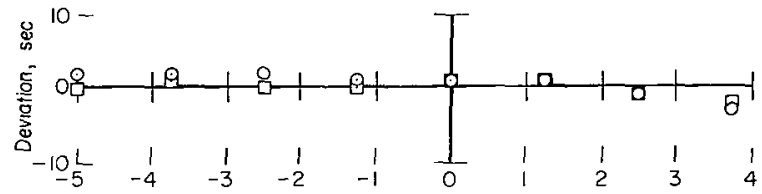

(b) Deviations perpendicular to plane of incidence for apertures in horizontal centerline.

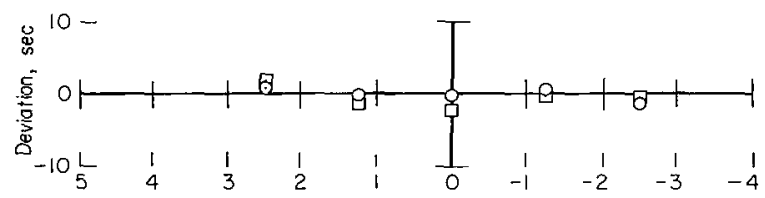

(c) Deviations in plane of incidence for apertures in vertical centerline.

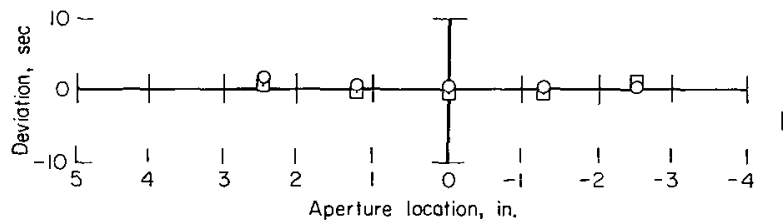

(d) Deviations perpendicular to plane of incidence for apertures in vertical centerline.

Figure 9.- Line-of-sight angular deviations through a spacecraft window at $45^{\circ}$ incidence.

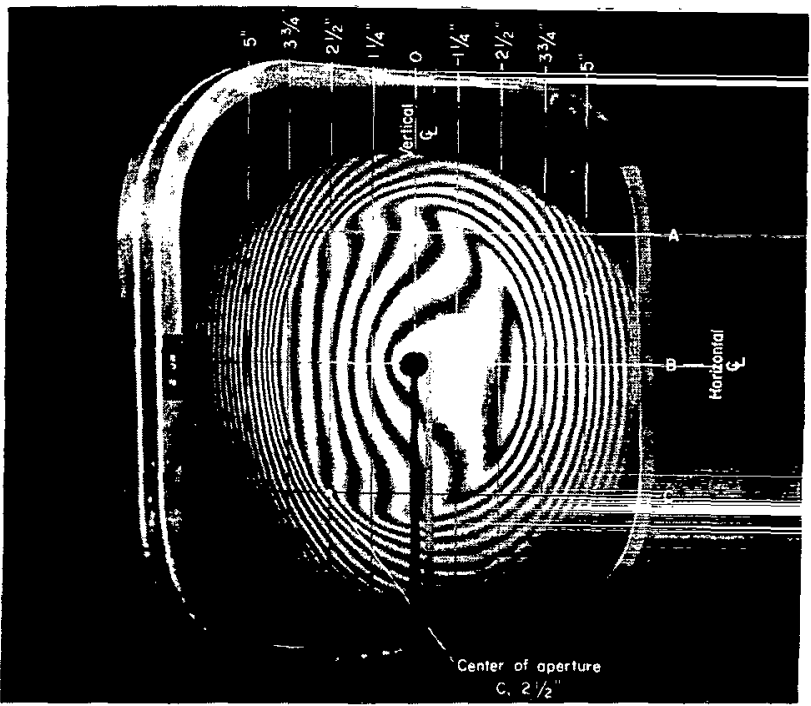

Figure 11. - Transmitted wave of aircraft window at $45^{\circ}$ incidence.

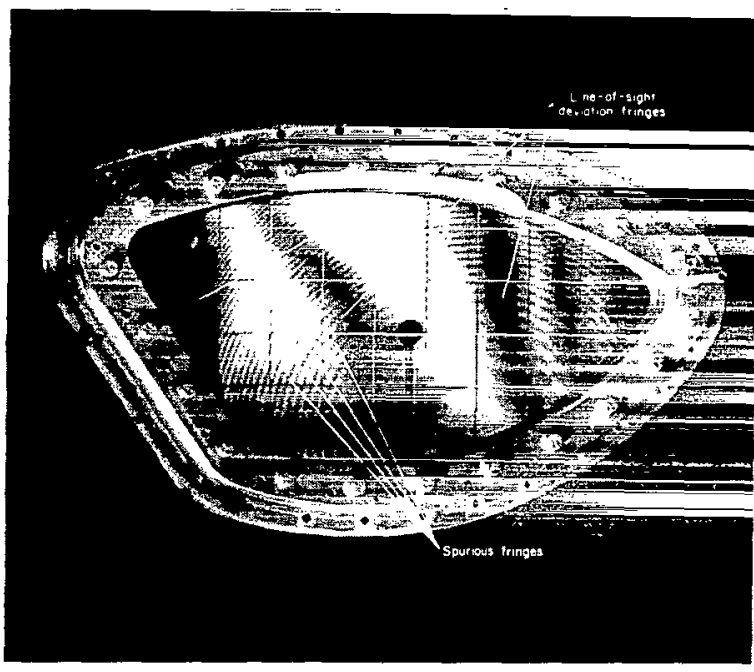

Figure 10.- Transmitted wave of spacecraft window at $45^{\circ}$ incidence.

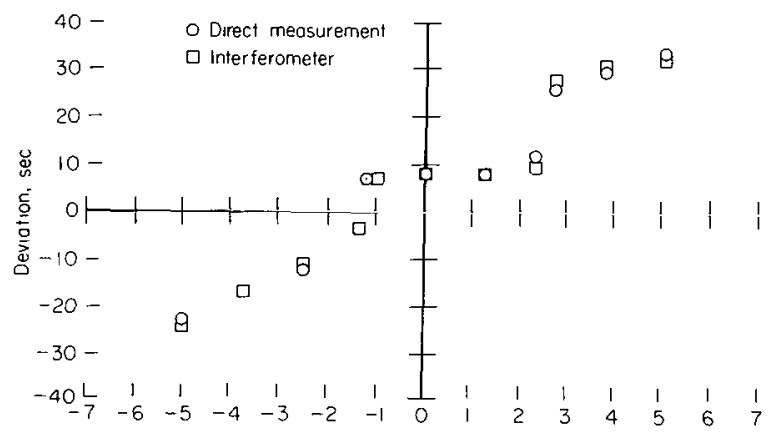

(a) Deviations in plane of incidence for apertures in row $C$.

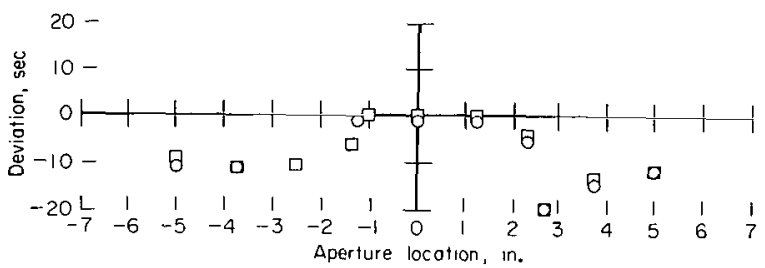

(b) Deviations perpendicular to plane of incidence for apertures in row $C$.

Figure 12.- Line-of-sight angular deviations through an aircraft window at $45^{\circ}$ incidence. 


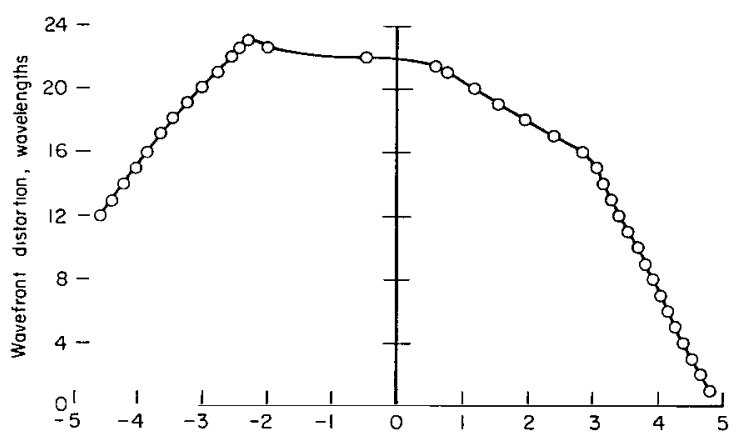

(a) Wavefront profile.

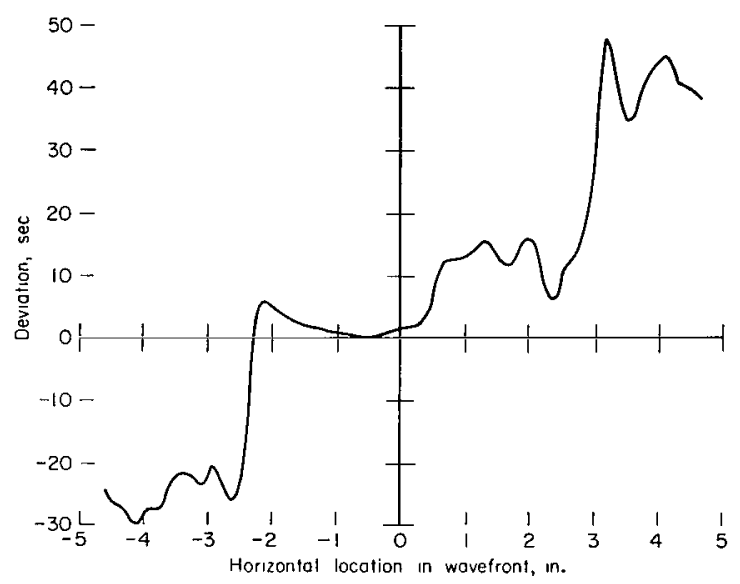

(b) Slope of wavefront profile.

Figure 13. - Wavefront profile and its slope at row $B$ of aircraft window for $45^{\circ}$ incidence in plane of row $B$.

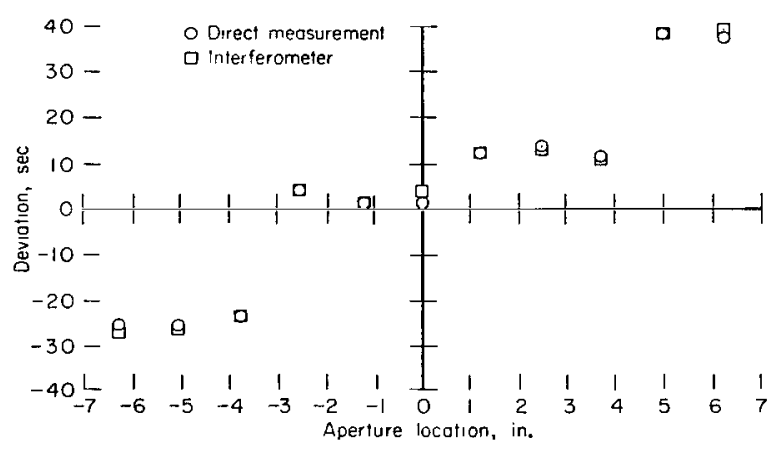

Figure 14.- Line-of-sight angular deviations in the plane of incidence for apertures in row $B$ of aircraft window at $45^{\circ}$ incidence.

4. In figure 11 , it can be seen that the deviations in row $B$ will lie principally in the horizontal plane of which $B$ is the trace. Because of this coincidence, the complete profile of the wavefront along row $B$ was plotted in figure 13(a). The fairly flat central portion and the steep slope on either side can be noted. The angular deviation is the first derivative or slope of this profile. This has been plotted in figure 13(b). The ixregularities of the slope represent actual deviations of the line of sight. In order to verify the data of figure 13, the mean deviation for apertures along row $B$ was plotted in figure 14, along with deviations obtained with the direct measurement apparatus. The measured and interferometric data agree in all cases to within 2 seconds of arc.

As a measure of the difference between the interferometric data and the measured data, the standard deviation of al1 these differences for the aircraft window data was only 0.7 second of arc, while that for the spacecraft window was just 0.6 second of arc.

Utilization of interference fringes for determining wavefront slope leads to consideration of Moiré fringes of the transmitted wave that would reveal patterns of constant slope, which, in turn, would correspond to patterns of constant angular deviations. Reference 9 touches on this subject of wavefront mapping by dual interferometry, and also shows what a Moiré fringe pattern is and what it reveals. Moiré patterns were obtained as part of this study, but the spacecraft window had so few fringes that the Moiré pattern was indistinct, and the graphical technique on a single interferogram proved to be a more sensitive data reduction method. The dual interferometer also required a fixed known wedge bias on both interferometers in known planes to keep the Moiré pattern slopes relative to the window axis. This 
compounded the data acquisition problem. Thus it was concluded that the Moiré pattern approach was unacceptable for high optical quality windows, and it was given no further consideration in this study.

\section{CONCLUSIONS}

The analysis of the interferograms as derived in the procedure proved to be a trustworthy and practical means for obtaining the deviations in seconds of arc. The interferometric method is particularly convenient because an interference photograph for a given test condition of incidence angle and plane of incidence yields errors for all apertures. The interference pattem must also be interrogated as in figure 6 to determine the type of wavefront (i.e., converging or diverging) to obtain the correct direction of deviation.

The precision obtainable with interferometry is excellent for determining angular deviations through a window. The standard deviation of the difference between interferometer data and precise directly measured data was 0.7 second of arc for the 129 measurements of this investigation. The range of deviations investigated - a few seconds to about 40 seconds - includes those of interest for navigational measurements in both spacecraft and aircraft.

Any conclusion on the relative merit of computed deviations versus interferometric data must be based on how valid the assumed boundary conditions are and on how reliable the glass characteristics of wedge, flatness, etc., are for the computational approach when balanced against the difficulties of obtaining the necessary interferograms. The interferometric experimental data, having all the anomalies of glass, frame, shell mounting, pressure deformation, etc. included in a transmitted wavefront, eliminated any error of omission or uncertainty.

The interferometry method limits the data to the particular angle of incidence and plane of incidence of the light entering the window. Thus, a separate interferogram is required for each of these parameter changes.

Deviation measurement systems, using either an autocollimator or an interferometric system, are faced with particular problems inherent in a test window, such as the duplication of environmental pressure, effects of the vehicle shell on the window mounting, and movement of the window or measurement apparatus to produce angles of incidence and planes of incidence of interest. The problems associated with mounting an autocollimator or interferometer adjoining a window of a spacecraft or aircraft are severe, particularly with regard to vibration and air movement. The feasibility of the interferometric system is dependent on the ability to adapt interferometric equipment more readily than the autocollimator to solve these problems. This 
study, while it did not attack all the practical problems involved, did prove that interferometric equipment is adaptable and has certain advantages over the autocollimator method.

Ames Research Center

National Aeronautics and Space Administration Moffett Field, Calif. 94035, June 3, 1969

\section{REFERENCES}

1. Smith, Donald W.; and Lampkin, Bedford A.: Sextant Sighting Measurements From On Board the Gemini XII Spacecraft. NASA TN D-4952, 1968.

2. Acken, Richard A.; and Smith, Donald W.: Navigator Performance Studies for Space Navigation Using the NASA CV-990 Research Aircraft. NASA TN D-4449, 1968.

3. Warner, David N., Jr.; and Walsh, Thomas M.: Effects of Edge Constraints on Optical Qualities of a Spacecraft Window. NASA TN D-4845, 1968.

4. Ashton, A.; and Marchant, A. C.: Note on Testing of Large Glass Panels. Optica Acta, vol. 14, no. 2, 1967, pp. 203-204.

5. Dyson, J.: Optics in a Hostile Environment. Applied Optics, vol. 7, no. 4, April 1968, pp. 569-580.

6. Stee1, W. H.: Interferometry. University Press, Cambridge, London, 1967.

7. Candler, C.: Modem Interferometers. Hilger and Watts, Ltd., London, Hilger Division, 1951.

8. White, Kenneth C.; and Gadeberg, Burnett L.: Methods for Predicting Spacecraft-Window-Induced Line-of-Sight Deviations. NASA TN D-5238, 1969.

9. Langenbeck, P.: Optical Wave-Front Mapping by Dual Interferometry . JOSA, vol. 58, no. 4, April 1968, pp. 499-505. 